\title{
Using Ultrafast X-ray Lasers to Image Structure \& Dynamics
}

\author{
Sébastien Boutet ${ }^{1}$
}

${ }^{1 .}$ Linac Coherent Light Source, SLAC National Accelerator Laboratory, Menlo Park, CA, USA

X-ray lasers have recently become a reality, with the Linac Coherent Light Source (LCLS) becoming the first ever X-ray Free Electron Laser (XFEL) in operation. The capabilities of XFELs are nothing short of revolutionary, with the production of coherent pulses of x-rays on the few femtosecond to few tens of femtosecond pulse duration regime, at wavelengths capable of achieving atomic resolution. These capabilities are opening the door to access new regimes in structural and dynamics studies with unprecedented simultaneous high temporal and spatial resolution.

Biological samples are known to be very sensitive to absorbed radiation which causes structural changes or damage to the sample during the exposure required to generate observable quantities about the sample. X-rays are typically absorbed more than they are scattered by roughly one order of magnitude. This implies that for techniques relying on the scattering of $\mathrm{x}$-rays such as many imaging techniques and crystal diffraction, the sample is inevitably damaged by the deposition of energy during the time needed for accumulation of the signal.

However, with ultrashort pulses of $\mathrm{x}$-ray, it is in principle possible to overcome the radiation damage limitations caused by long, integrating measurements. If the pulses are short enough to allow all incident $\mathrm{x}$-rays to pass through the sample faster than the response of the atoms to the absorbed energy, it is possible to obtain structural information free of damage [1]. This technique is now known as "diffraction-then-destruction", where significantly larger doses can be absorbed by the sample than what is possible with continuous sources of x-rays, while still producing interpretable signal, due to the fact that the inertia in the sample causes the radiation induced changes in the sample to propagate slower than the pulse duration. These assumptions and damage models can now be tested at LCLS.

The diffraction-before-destruction technique using x-rays has been demonstrated to be a valuable tool to study a variety of samples using a few different techniques, including crystallography [2-4], diffractive imaging [5] and various spectroscopic techniques [6].

Serial Femtosecond Crystallography (SFX) [2], a new technique which uses a constant flow of small protein crystals inside a liquid jet, has been demonstrated to yield damage-free high-resolution structures. It has since been utilized to study increasingly challenging samples that have escaped structural determination using other techniques. New biological information can now be obtained using this technique $[3,4]$. The typical experimental geometry is shown on Figure 1. Snapshots of the samples are taken with the short x-ray pulses without any sample motion during the pulse. This allows the study of dynamics if a structural change can be initiated in the sample using some trigger with a tunable delay. The addition of a laser illuminating the sample prior to the arrival of x-rays can be used as one such trigger and makes accessible ultrafast dynamics. The technique of time-resolved Serial Femtosecond Crystallography has been demonstrated [7] and opens the door to making molecular movies of important reactions such as those involved in photosynthesis.

Coherent Diffractive Imaging (CDI) is a technique which measures the continuous diffraction patterns 
of single objects and utilizes computational phase retrieval methods to recover an image of the sample. The technique has been under development for about 15 years and holds the possibility of studying single particles at high resolution without the need to crystallize them when used in combination with an X-ray Free Electron Laser like LCLS. Single particle imaging has been demonstrated with limited spatial resolution [5] and still requires development work to become a useful tool for scientific discovery but if successful, would open the doors to unique possibilities to study structural dynamics of single molecules.

Overall, structural and dynamics studies enabled by LCLS are numerous in a verity of fields. Structural biology has been an early area of broad activity and exciting results have been and will continue to be obtained.

References:

[1] Neutze, R., et al., Nature 406 (2000), p. 752-757.

[2] Boutet, S., et al., Science 337 (2012), p. 362-364.

[3] Liu, W., et al., Science 342 (2013), p. 1521-1524.

[4] Redecke, L., et al., Science 339 (2013), p. 227-230.

[5] Seibert, M.M., et al., Nature 470 (2011), p. 78-U86.

[6] Alonso-Mori, R., et al., PNAS 109 (2012), p. 19103-19107.

[7] Tenboer, J., et al., Science 346 (2014), p. 1242-1246.

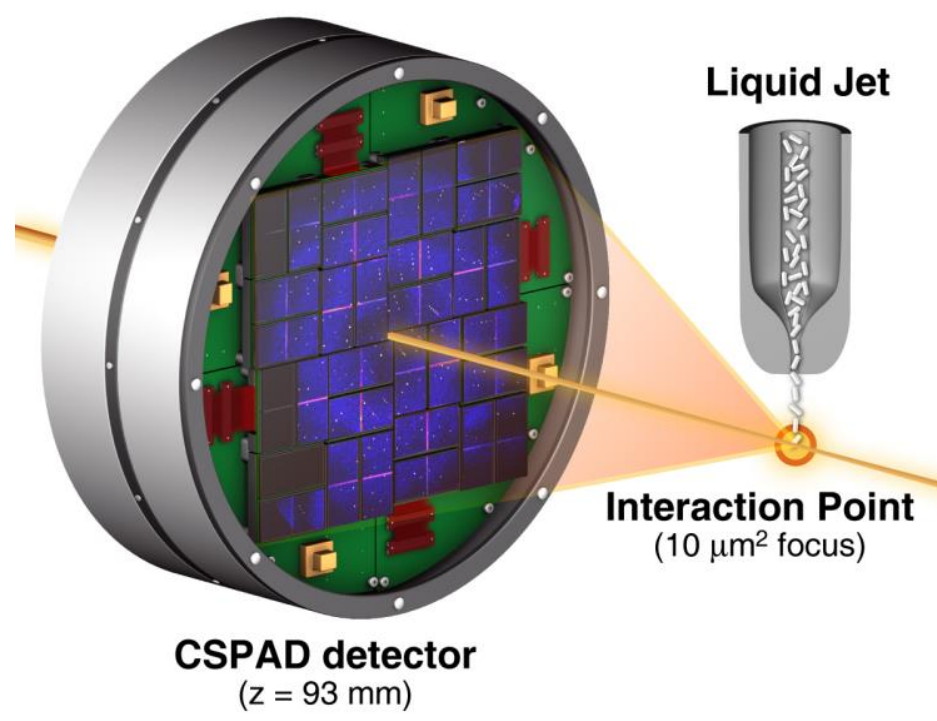

KB Mirrors $(z=93 \mathrm{~mm})$

Undulator

(420 m upstream)

Figure 1. Experimental geometry for serial femtosecond crystallography (SFX). 